\title{
Rotationally Symmetric Lacunary Functions and Products of Centered Polygonal Lacunary Functions
}

\author{
L. K. Mork ${ }^{1}$ (D), Keith Sullivan ${ }^{1}$, Trenton Vogt ${ }^{2}$ and Darin J. Ulness ${ }^{2, * \mathbb{D}}$ \\ 1 Department of Mathematics, Concordia College, Moorhead, MN 56562, USA; lmork@cord.edu (L.K.M.); \\ ksulliv1@cord.edu (K.S.) \\ 2 Department of Chemistry, Concordia College, Moorhead, MN 56562, USA; tvogt@cord.edu \\ * Correspondence: ulnessd@cord.edu
}

Received: 30 March 2020; Accepted: 22 May 2020; Published: 26 May 2020

\begin{abstract}
This work builds upon previous studies of centered polygonal lacunary functions by presenting proofs of theorems showing how rotational and dihedral mirror symmetry manifest in these lacunary functions at the modulus level. These theorems then provide a general framework for constructing other lacunary functions that exhibit the same symmetries. These investigations enable one to better explore the effects of the gap behavior on the qualitative features of the associated lacunary functions. Further, two renormalized products of centered polygonal lacunary functions are defined and a connection to Ramanunjan's triangular lacunary series is made via several theorems.
\end{abstract}

Keywords: lacunary function; gap function; centered polygonal numbers; natural boundary; singularities; renormalization

\section{Introduction}

Without a doubt, complex analytic functions are both a rich and beautiful area of mathematical pursuit and one of the most powerful tools used in physics to gain insight into countless natural phenomena. One important reason for this is that singularities- the points where analyticity breaks down - carry physical information about the system. The most immediate example is the damped simple harmonic oscillator, where the position of the associated pole in the complex plane gives the natural frequency and damping constant. Analytic functions can be represented by Taylor series, the radius of convergence of which are restricted by singular points. For the ubiquitous case of functions having only isolated singularities, analytic continuation can be performed to expand the domain of the function throughout the complex plane (or, more generally, Riemann sheet).

A much less frequently explored class of analytic functions are those with singular curves called natural boundaries, which are impenetrable to analytic continuation [1,2]. Of particular note are the lacunary functions. These are functions that exhibit a natural boundary and whose power series has absent terms-"gaps" or "lacunae"-in the progression of the exponential powers within the expansion. One simple example is $f(z)=\sum_{n=1}^{\infty} z^{n !}=z+z^{2}+z^{6}+z^{24}+\cdots$, which has a natural boundary on the unit circle. The function is analytic in the open unit disk and cannot be analytically continued beyond the unit circle. Figure 1 shows a graphical representation of the modulus of this function. The lower right panel representation, which shows a contour plot of $|f(z)| \leq 1$, is used throughout this work.

In general, Hadamard's gap theorem establishes the criterion for the presence of a natural boundary of series with gaps [2]. The lacunary functions explored in this work meet this criterion because the gaps in the exponential powers increase as the series expansion goes to infinity.

Due to analytic continuation not being possible through the natural boundary as well as additional complications, functions with natural boundaries have not often seen use in physics. Recently, however, 
lacunary functions have seen use in addressing physical problems. Natural boundaries influencing instanton orbiting was shown by Shudo and Ikeda to impact quantum tunneling [3]. Creagh and White demonstrated the importance of natural boundaries in the short-wavelength approximation when calculating evanescent waves outside of elliptic dielectrics [4]. Lacunary functions are related to Gauss sums, of which Schleich et al. discussed the many applications in physics [5,6]. Regarding integrable/nonintegrable systems, Greene and Percival worked with natural boundaries in their study of Hamiltonian maps [7].

In statistical mechanics, Nickel proved that, within the calculation of the magnetic susceptibility in the 2D Ising model, there is the presence of a natural boundary [8]. Additionally, several research groups have shown that any solution of an unsolvable Ising-like system must be able to be expressed in terms of functions having natural boundaries $[9,10]$. In kinetic theory, lacunary functions, upon approaching the natural boundary, have features that are closely tied to Weiner (stochastic) processes. Because of this, lacunary functions have been discussed in the context of Brownian motion [2,11]. In quantum mechanics, Yamada and Ikeda examined the wavefunctions related to Anderson-localized states in the Harper model [12].

Alongside physics, lacunary functions have found use in probability theory. Particular lacunary trigonometric systems $[13,14]$ work as independent random variables. Criteria for the behavior of the gaps in order for these lacunary functions to be consistent with the central limit theorem have been established by several researchers [13,15-17]. Lacunary trigonometric systems are also of interest in the study of harmonic analysis on compact groups [18,19]. Costin and Huang provided a detailed study of the behavior of lacunary series at the natural boundary [20]. Very recently, Boyd investigated the breakdown of the Darboux principle at the natural boundary of lacunary function [21]. Dahlqvist studied conditions of the zeta function that produce natural boundaries [22]. Additionally, lacunary functions are useful in partitioning theory [23-26]. Further, lacunary series have been discussed with their relationship to theta, mock theta, and Dedekind eta functions [27,28]. Tangentially related to the current work is the very recent study of Bohr's inequality via lacunary series by Kayumov and Ponnusamy [29] and Liu and Liu [30]. Eckstein and Zając studied heat traces of unbounded operators in Hilbert space [31]. In their investigation of Sobolev-Jacobo polynomials, Behr et.al. studied lacunary generation functions [32]. Kişi, Gümüş, and Savas investigated $A^{\mathcal{I}}$-lacunary convergence and Cesàro summability with respect to lacunary sequences [33].

Recent work of the current authors has focused on centered polygonal lacunary functions [34-37]. The exponential power gaps for these functions are given by the centered polygonal numbers [38,39]. The modulus of centered polygonal lacunary functions exhibits rotational symmetry under a phase shift in the complex plane. This is related to the fact that lacunary functions also exhibit well organized behavior at the natural boundary [34]. Most lacunary functions exhibit what is called quasi-symmetry [34] but not true rotational symmetry. They also have fractal character [36]. Insight from the study has allowed for an understanding of what must be true of the gap behavior in order for the lacunary function to exhibit true rotational symmetry as well as dihedral mirror symmetry. These symmetries are the topic of this work and the subject of four theorems presented below. Several examples are presented as is a discussion of the nature of the gap behavior and its effect on the form of the lacunary functions.

A second aspect of this paper is to develop two distinct renormalized products of centered polygonal lacunary functions which carry with them the rotational and dihedral mirror symmetry of the centered polygonal lacunary functions. The first renormalized product is simply a power of a single centered polygonal lacunary function. The second is a product of symmetry-related centered polygonal lacunary functions. 


\section{Definitions and Notation}

The Nth member of a lacunary sequence of functions is given by

$$
f_{N}(z)=\sum_{n=1}^{N} z^{g(n)},
$$

where the function $g(n)$ satisfies Hadamard's gap theorem [2]. Note that the sum starts at $n=1$ rather than at $n=0$ [34]. Further, to limit the scope of this work, only the cases where $g(1)=1$ and unit coefficients are considered. For convenience, Haramard's theorem is repeated here as given in [2].

Theorem 1. Hadamard's Gap Theorem. The analytic function defined by $f(z)=\sum_{n=1}^{\infty} z^{g(n)}$ has a natural boundary on the boundary of its radius of convergence if there exists a fixed $\lambda>1$ such that, for all $n$

$$
\frac{g(n+1)}{g(n)} \geq \lambda
$$

For most of what follows. $g(n)$ is some sort of quadratic function. Consider the prototypical example of $g(n)=n^{2}$. In this case, $\frac{(n+1)^{2}}{n^{2}}>1$ for all $n$ so there exists a fixed $\lambda>1$ such that $\frac{(n+1)^{2}}{n^{2}} \geq \lambda$.

For convenience of notation. one defines

$$
\mathfrak{L}(g ; z) \equiv\left\{\sum_{n=1}^{N} z^{g(n)}\right\},
$$

to represent the particular lacunary sequence described by $g(n)$. The condensed notation, $f_{N}(z)$, is used throughout to represent the $N$ th member of $\mathfrak{L}(g ; z)$. The lacunary function associated with the sequence is defined in the limit,

$$
\lim _{N \rightarrow \infty} f_{N}(z) \equiv f(z)
$$

The example from Section 1 would be

$$
\mathfrak{L}(n ! ; z) \equiv\left\{\sum_{n=1}^{N} z^{n !}\right\},
$$

and the specific function shown in Figure 1 is $f_{40}(z)$. Finally, it is noted that there are $N$ terms in the summations and that the sum truncates after the $g(N)$ th power, thus for $f_{40}(z)$ the highest power in the sum is $z^{40 !}$.

As a matter of convenience for the reader, several definitions and theorems regarding rotational symmetry are collected here. Proofs and other discussion can be found in [34].

Definition 1. Centered polygonal number. The formula for the set of centered $k$-gonal numbers is

$$
C^{(k)}=\left\{k \frac{n(n-1)}{2}+1\right\}, \quad n \geq 1
$$

Centered polygonal numbers are an infinite, increasing sequence of numbers associated with points on a polygonal lattice [38-41]. Teo and Sloane [39] discussed centered polygonal numbers in the context of two-dimensional crystal structures. The centered polygonal numbers are related to the more familiar polygonal numbers, $P^{(k)}[40,41]$. Note $T \equiv P^{(3)}$ are the ubiquitous triangular numbers.

Lemma 1. $P^{(k)}(n)+(n-1)^{2}=C^{(k)}(n)$.

Proof. The proof is given in [34]. 
For the special case of triangular numbers,

\section{Lemma 2.}

$$
\frac{C^{(k)}(n+1)-1}{k}=T(n) .
$$

Proof. The proof is given in [34].

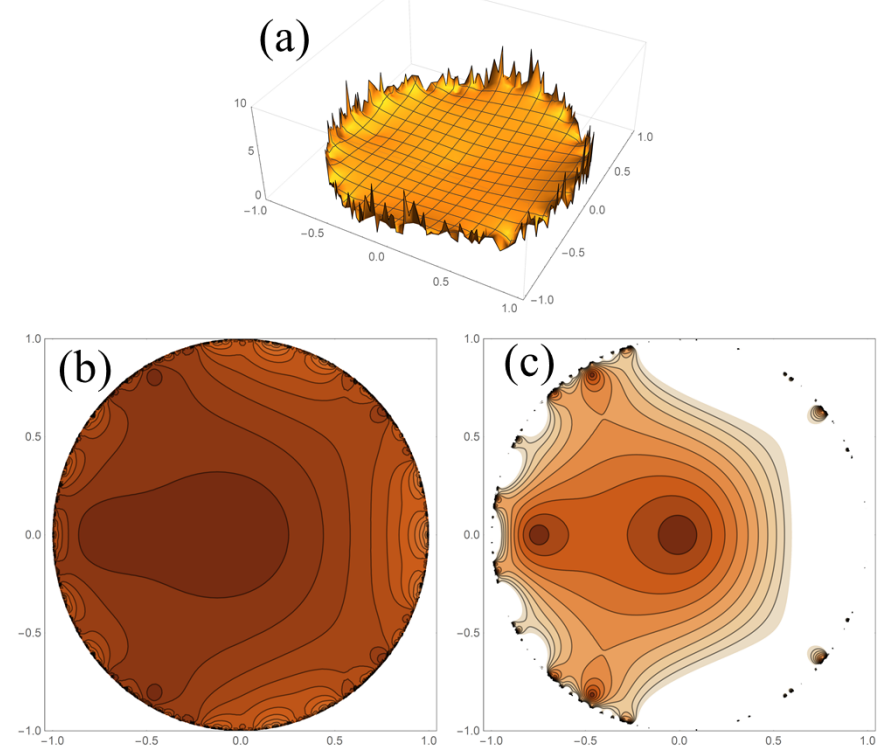

Figure 1. The example of $f(z)=\sum_{n=1}^{\infty} z^{n !}$. To render the plot an upper limit, $N$, on the sum must be chosen. In this case, $N=40$. Panel (a) is the three-dimensional graph of $|f(z)|$. Panel (b) shows the corresponding contour plot. Panel (c) is a particularly important representation. In this case, the contour plot is limited to $|f(z)| \leq 1$. The contour at $|f(z)|=1$ is referred to as the unity contour. The part of the unity contour which is a closed curve encircling the origin is called the main unity contour (see Definition 2).

When it comes to the source for $g(n)$, centered polygonal numbers give rise to lacunary functions that exhibit true rotational symmetry along with dihedral mirror symmetry. The polygonal numbers do not.

Definition 2. Main unity contour. The simple closed rectifiable curve marking the boundary of the set of points, $z$, such that $|f(z)| \leq 1$ and are path-connected to the origin.

Definition 3. Primary symmetry. The rotational symmetry of the $N=2$ member of $|\mathfrak{L}(g ; z)|,\left|f_{2}(z)\right|$, is called the primary symmetry.

Theorem 2. The primary symmetry of $|\mathfrak{L}(g(n) ; z)|$ is $s \equiv g(2)-g(1)$.

Proof. The proof is given in [34].

Corollary 1. The primary symmetry of the centered polygonal lacunary functions $\left(g(n)=C^{(k)}(n)\right)$ is $k$, i.e., $s=k$.

Proof. The proof is given in [34]. 
Definition 4. Symmetry angle. Let the primary symmetry be s-fold. The first symmetry angle is $\alpha_{1}=\frac{\pi}{s}$, $s \in \mathbb{Z}^{+}$. The pth symmetry angle is $\alpha_{p}=\frac{\pi}{p s}, p, s \in \mathbb{Z}^{+}$.

\section{Rotational and Dihedral Symmetry of Centered Polygonal Lacunary Functions}

Turning attention to the centered polygonal lacunary functions, one sees clear $k$-fold rotational symmetry. The examples of $k=3, k=5$, and $k=7$ are shown in Figure 2 which have three-, five-, and seven-fold true rotational symmetry, respectively. In addition to the rotational symmetry, $k$ dihedral mirror planes of symmetry exist. These appear at multiples of $\alpha_{1}$, as clearly seen in Figure 2 . The proof of the existence of these symmetries is straightforward. The following two theorems establish these symmetries. When considering centered polygonal lacunary functions, $f_{N}(z)$ is written as $f_{N}^{(k)}(z)$ to expose the $k$ parameter.
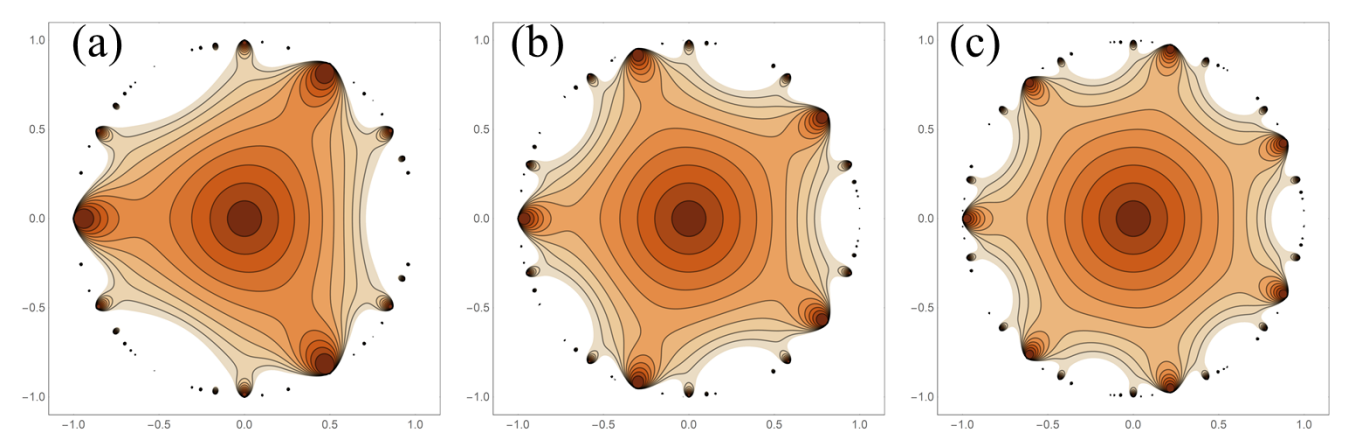

Figure 2. The example of lacunary functions generated from the centered polygonal numbers. Panel (a) shows the case for $k=3, N=20$. Panel (b) shows the case for $k=5, N=20$. Panel (c) shows case for $k=7, N=20$. In all cases, the true rotational symmetry and dihedral mirror symmetry are clearly exposed.

Theorem 3. The functions in the set $\mathfrak{L}\left(C^{(k)}(n) ; z\right)$ have k-fold rotational symmetry at the modulus level, where $k$ is any positive integer.

Proof. The complex variable $z$ is represented in polar form, $z=\rho e^{i \phi}$. Writing out the $N$ th member of the lacunary sequence $\mathfrak{L}\left(C^{(k)}(n) ; \rho e^{i \phi}\right)$, one has

$$
\begin{aligned}
f_{N}^{(k)}(z) & =\sum_{n=1}^{N} z^{C^{(k)}(n)}=\sum_{n=1}^{N} \rho^{C^{(k)}(n)} e^{i C^{(k)}(n) \phi} \\
& =\sum_{n=1}^{N} \rho^{C^{(k)}(n)} e^{i \frac{k n^{2}-k n+2}{2} \phi}=\sum_{n=1}^{N} \rho^{C^{(k)}(n)} e^{i k \frac{n(n-1)}{2} \phi} e^{i \phi} \\
& =\sum_{n=1}^{N} \rho^{C^{(k)}(n)} e^{i k T(n-1) \phi} e^{i \phi} \equiv f_{N}(\phi) .
\end{aligned}
$$


Now, consider $f_{N}^{(k)}\left(\phi+\frac{2 \pi}{k}\right)$,

$$
\begin{aligned}
f_{N}^{(k)}\left(\phi+\frac{2 \pi}{k}\right) & =\sum_{n=1}^{N} \rho^{C^{(k)}(n)} e^{i k T(n-1)\left(\phi+\frac{2 \pi}{k}\right)} e^{i\left(\phi+\frac{2 \pi}{k}\right)} \\
& =\sum_{n=1}^{N} \rho^{C^{(k)}(n)} e^{i k T(n-1) \phi} e^{i \phi} e^{i 2 \pi T(n-1)} e^{i \frac{\pi}{k}} \\
& =e^{i \frac{i}{k}} \sum_{n=1}^{N} \rho^{C^{(k)}(n)} e^{i k T(n-1) \phi} e^{i \phi} .
\end{aligned}
$$

Upon evaluating the modulus, $\left|f_{N}^{(k)}\left(\phi+\frac{2 \pi}{k}\right)\right|=\left|f_{N}^{(k)}(\phi)\right|$. Hence, $k$-fold rotational symmetry is present and evident in the unity contour plots.

Theorem 4. The functions in the set $\mathfrak{L}\left(C^{(k)}(n) ; z\right)$ have dihedral mirror symmetry at the primary symmetry angles at the modulus level (see Figure 2).

Proof. First, consider

$$
\begin{aligned}
f_{N}^{(k)}\left(\phi+\alpha_{1}\right) & =\sum_{n=1}^{N} \rho^{C^{(k)}(n)} e^{i C^{(k)}(n) \phi} e^{i C^{(k)}(n) \alpha_{1}} \\
& =\sum_{n=1}^{N} \rho^{C^{(k)}(n)} e^{i C^{(k)}(n) \phi} e^{i \frac{n(n-1)}{2} \pi} e^{i \frac{\pi}{k}}
\end{aligned}
$$

Recognizing $e^{i \frac{n(n-1)}{2} \pi}$ as $(-1)^{T(n-1)}$, one has

$$
f_{N}^{(k)}\left(\phi+\alpha_{1}\right)=e^{i \frac{\pi}{k}} \sum_{n=1}^{N}(-1)^{T(n-1)} \rho^{C^{(k)}(n)} e^{i C^{(k)}(n) \phi} .
$$

Now, consider the mirror reflection about the line at $\alpha_{1}$,

$$
f_{N}^{(k)}\left(-\phi+\alpha_{1}\right)=e^{i \frac{\pi}{k}} \sum_{n=1}^{N}(-1)^{T(n-1)} \rho^{C^{(k)}(n)} e^{-i C^{(k)}(n) \phi} .
$$

Thus, $f_{N}^{(k)}\left(\phi+\alpha_{1}\right)$ is of the form $e^{i \frac{\pi}{k}} u(\phi)$ and $f_{N}^{(k)}\left(-\phi+\alpha_{1}\right)$ is of the form $e^{i \frac{\pi}{k}} u^{*}(\phi)$. These two expressions are equivalent under the modulus $\left|f_{N}^{(k)}\left(\phi+\alpha_{1}\right)\right|=\left|f_{N}^{(k)}\left(-\phi+\alpha_{1}\right)\right|$, thus proving the dihedral mirror symmetry at the modulus level.

\section{Constructing Rotationally Symmetric Lacunary Functions}

The case of the centered polygonal lacunary functions provides insight into what must be true of the behavior of the gaps in the power series. In the above proofs, one notices the cancellation of the $k$ for all the $n$-dependent phase factors. If this is true, then the lacuanary function will have true rotational symmetry and dihedral mirror symmetry at the modulus level.

This insight leads to a method to construct lacunary functions that exhibit rotational and dihedral mirror symmetry. The main idea is that $g(n)$ must be chosen as such to produce the same rotational symmetry as the primary symmetry. To aid in thinking, consider $g(n)=s \cdot h(n)+1$, with $h(n)$ chosen as follows: Choose an $h(n)$ which gives rise to an infinite, strictly increasing, superlinear sequence such that the first two members of the series are 0 and 1, i.e., $h(1)=0$ and $h(2)=1$. This will yield a primary symmetry (and hence quasi-symmetry) of $g(2)-g(1)=s$, hence $\alpha_{1}=\frac{\pi}{s}$. Further, the $n$-dependent part of $g(n)$ is homogeneous and linear in $s$. This will allow the $s$ to cancel out of the phase factors similar to in the proofs in Section 3. The above conditions on $h(n)$ can be loosened such that it only 
matters that the first two values of the sequence generated by $h(n)$ differ by one. The more restricted version is used in this work to ensure the first power of $z$ is present in the power series.

The above ideas can be collected into general symmetry theorems as follows.

Theorem 5. Let $s$ and $n$ be positive integers. The members of $\mathfrak{L}(g ; z)$ have s-fold rotational symmetry at the modulus level when $g(n)=s \cdot h(n)+1$.

Proof. The proof follows that of Theorem 3. Writing out the $N$ th member of the lacunary sequence $\mathfrak{L}\left(g(n) ; \rho e^{i \phi}\right)$, one has

$$
\begin{aligned}
f_{N}^{(s)}(z) & =\sum_{n=1}^{N} z^{g(n)}=\sum_{n=1}^{N} \rho^{g(n)} e^{i(s \cdot h(n)+1) \phi} \\
& =e^{i \phi} \sum_{n=1}^{N} \rho^{g(n)} e^{i s \cdot h(n) \phi} \equiv f_{N}^{(s)}(\phi) .
\end{aligned}
$$

Now, consider $f_{N}^{(s)}\left(\phi+\frac{2 \pi}{s}\right)$,

$$
\begin{aligned}
f_{N}^{(s)}\left(\phi+\frac{2 \pi}{s}\right) & =e^{i \phi+\frac{2 \pi i}{s}} \sum_{n=1}^{N} \rho^{g(n)} e^{i s \cdot h(n)\left(\phi+\frac{2 \pi}{s}\right)} \\
& =e^{i \phi+\frac{2 \pi i}{s}} \sum_{n=1}^{N} \rho^{g(n)} e^{i s \cdot h(n) \phi} e^{i h(n) 2 \pi} \\
& =e^{\frac{2 \pi i}{s}} e^{i \phi} \sum_{n=1}^{N} \rho^{g(n)} e^{i s \cdot h(n) \phi}=e^{\frac{2 \pi i}{s}} f_{N}^{(s)}(\phi) .
\end{aligned}
$$

Thus, at the modulus level, $\left|f_{N}^{(s)}\left(\phi+\frac{2 \pi}{s}\right)\right|=\left|f_{N}^{(s)}(\phi)\right|$.

Notice that, although the scope of this work considers only the cases where $h(1)=0$ and $h(2)=1$, Theorem 5 does not require that restriction. The dihedral mirror symmetry follows similarly.

Theorem 6. The members of $\mathfrak{L}(g ; z)$ have dihedral mirror symmetry along multiples of $\frac{\pi}{s}$ at the modulus level when $g(n)=s \cdot h(n)+1$.

Proof. Similar to how the proof of Theorem 5 follows that of Theorem 3, so does the proof of this theorem follow that of Theorem 4.

$$
\begin{aligned}
f_{N}^{(s)}\left(\phi+\frac{\pi}{s}\right) & =\sum_{n=1}^{N} \rho^{g(n)} e^{i g(n) \phi} e^{i g(n) \frac{\pi}{s}} \\
& =e^{i \frac{\pi}{s}} \sum_{n=1}^{N} \rho^{g(n)} e^{i g(n) \phi} e^{i h(n) \pi}
\end{aligned}
$$

Now,

$$
\begin{aligned}
f_{N}^{(s)}\left(-\phi+\frac{\pi}{s}\right) & =\sum_{n=1}^{N} \rho^{g(n)} e^{-i g(n) \phi} e^{i g(n) \frac{\pi}{s}} \\
& =e^{i \frac{\pi}{s}} \sum_{n=1}^{N} \rho^{g(n)} e^{-i g(n) \phi} e^{i h(n) \pi} .
\end{aligned}
$$

Since $e^{i h(n) \pi}$ is real, $\left|f_{N}^{(s)}\left(\phi+\frac{\pi}{s}\right)\right|=\left|f_{N}^{(s)}\left(-\phi+\frac{\pi}{s}\right)\right|$, as it is for the similar case of Theorem 4. Thus, there exist dihedral mirror symmetry at $\frac{\pi}{s}$. 
By way of concrete example, the Fibonacci numbers are first considered: $\{\mathrm{fib}(n)\}=$ $\{0,1,1,2,3,5,8,13,21, \ldots\}$. At first glance, it looks like this is a good candidate for an interesting $h(n)$. However, the presence of two consecutive 1 s does not make $h(n)$ strictly increasing. The two consecutive $1 \mathrm{~s}$ will result in a power series where the coefficient of the $z^{s+1}$ term is 2 , not 1 as required. The scope of this paper is for lacunary functions of the form of Equation (1). (The generalization to non-unity coefficients is an important one and should be a fertile ground for future research.) For completeness, this function is plotted in Figure 3 where it does have rotational and mirror symmetry.

To get the Fibonacci sequence into a form that fits within the scope of this work, one can consider $h(n)=\mathrm{fib}(n+1)-1$, Thus, the first two members of the sequence generated by $h(n)$ are 0 and 1 as required and the sequence is superlinear and strictly increasing. The resultant lacunary functions is shown in Figure 3b,c. Both symmetries are clearly evident. It is interesting to note how different the main unity contour plot is when compared to the directly-used Fibonacci sequence.
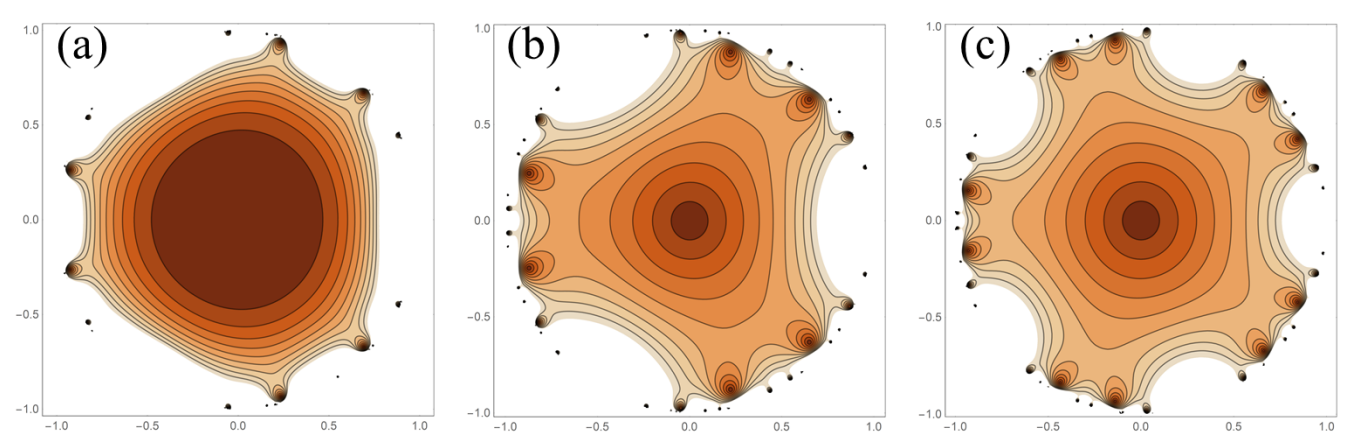

Figure 3. The example of lacunary functions generated from the Fibonacci sequence. Panel (a) shows the case for $s=3, N=20$, and $h(n)=$ fib $(n)$. Panel $(\mathbf{b})$ shows the case for $s=3, N=20$, and $h(n)=$ fib $(n+1)-1$. Panel (c) shows the same case as the middle panel but now $s=5$.

Sequences given by powers give rise to qualitatively different lacunary functions than those arising from the Fibonacci sequences. Figure 4 shows the lacunary functions generated when $h(n)=(n-1)^{2}$, $h(n)=(n-1)^{4}$, and $h(n)=(n-1)^{8}$. These sequences "accelerate" faster than the Fibonacci sequence in that the early numbers following the first 0 and 1 are much larger for the powers. However, the Fibonacci sequence ultimately surpasses the powers. The most notable qualitative distinction between the Fibonacci-based and power-based lacunary functions is the fact that the set bounded by the main unity contour is nearly convex for the power-based lacunary functions but that certainly is not the case for the Fibonacci-based lacunary functions. The manner in which $g(n)$ behaves has a significant influence on the qualitative shape of the main unity contour.

A more systematic study of the effect of the rapidness of the increase of the gap can be made by defining a function $h(n)=\left\lfloor(n-1)+\frac{(n-1)^{2}}{w}\right\rfloor$ where $\lfloor x\rfloor$ is the floor of $x$. The "weight" parameter, $w$, softens the rising values of the sequence generated by $h(n)$. The associated lacunary functions for several values of $w$ are shown in Figure 5. As $w$ increases, qualitative changes to the associated lacunary functions are seen. This is especially true for low values of $w$. As $w$ increases, the qualitative shape of the associated lacunary function settles into a single form with changes occurring only very near the natural boundary. As the rate of increase of the gap goes down, so does the convexity of the domain contained in the main unity contour. 

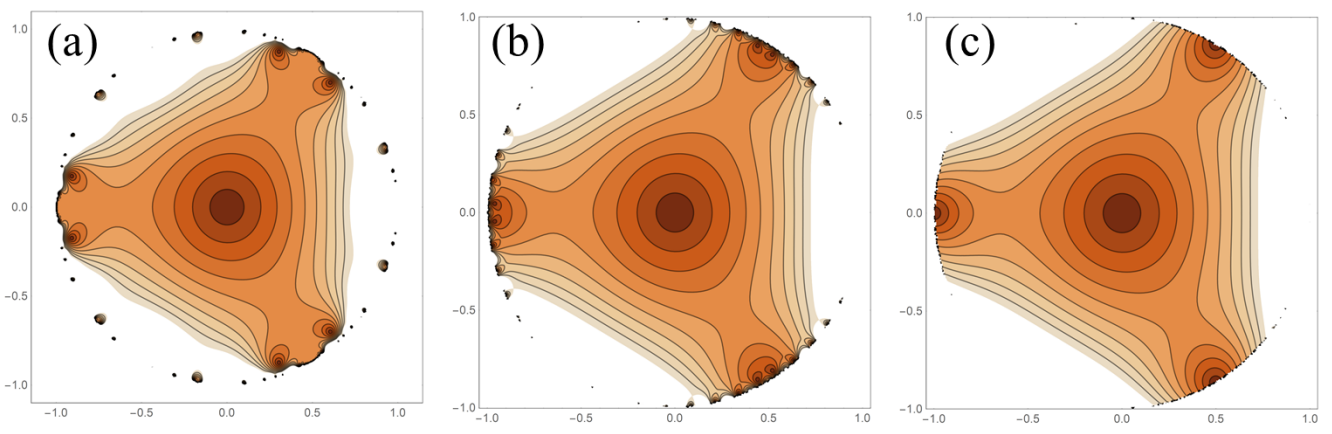

Figure 4. The example of lacunary functions generated from the powers of $n$. In all cases $s=3$ and $N=20$. Panel (a) shows $h(n)=(n-1)^{2}$. Panel (b) shows the case for $h(n)=(n-1)^{4}$. Panel (c) shows the case for $h(n)=(n-1)^{8}$. Notice the qualitative similarity among these but the qualitative distinctiveness compared with the lacunary functions generated from the Fibonacci sequence. This is because the power sequence "accelerates" faster than the Fibonacci sequence does, even though the rate of increase ultimately favors the Fibonacci sequence.
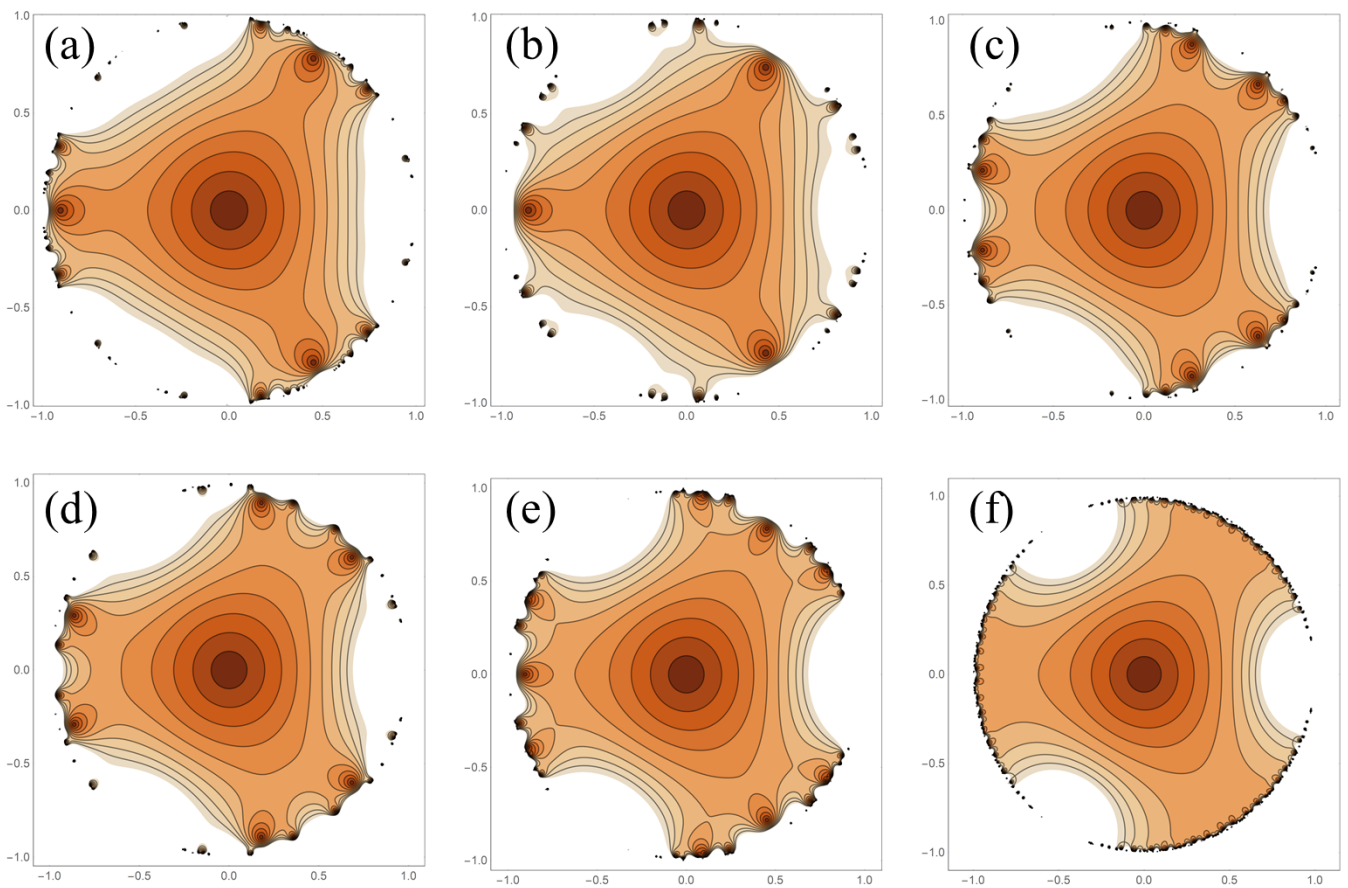

Figure 5. A sequence of related lacunary functions that systematically vary the rate of gap increase. Here, $h(n)=\left\lfloor(n-1)+\frac{(n-1)^{2}}{w}\right\rfloor$. In all cases, $k=3$ and $N=200$. Across the top row from left to right: (a) $w=1,(\mathbf{b}) w=3$, and (c) $w=6$. Across the bottom row from left to right: (d) $w=8,(\mathbf{e}) w=9$, and (f) $w=500$. At low values of $w$ (rapid growth of the gap), one sees significant qualitative changes in the graphs for modest changes in $w$. At higher values of $w$, the qualitative features of the graph stabilize. There is a general trend from higher convexity of the domain bounded by the the main unity contour to lower convexity. (Note: a very small positive value $\left(1 \times 10^{-6}\right)$ is added to $w$ during the rendering to ensure $h(1)=1$ when $w=1$.)

Comparing Figures 5,3, and 4, one sees that, for small $w$, the main unity contour is qualitatively similar to that for the power-based lacunary functions (Figure 4). Conversely, for large $w$, there is qualitative similarity with the Fibonacci-based lacunary function (Figure 3). Of course, in both cases, 
there are numerous differences as well (especially near the natural boundary). This points to the sensitivity of the lacunary functions to the behavior of $g(n)$.

\section{Renormalized Products of Centered Polygonal Lacunary Functions}

Having studied the rotational and mirror symmetry of certain lacunary functions, attention is returned to centered polygonal lacunary functions and, specifically, to the behavior of their products. These products also exhibit the same symmetries at the modulus level. Additionally, the notion of convexity of the main unity contour discussed above is also at play with the products.

The triangular lacunary series of Ramanujan is [24,42],

$$
\psi(q):=\sum_{j=0}^{\infty} q^{\frac{j(j+1)}{2}}=\sum_{n=0}^{\infty} q^{T(j)},
$$

where $T(j)$ represents the $j$ th triangular number, which is well-known in partition theory [42]. The $m$-fold product,

$$
\psi(q)^{m}=\sum_{a=0}^{\infty} \sum_{b=0}^{\infty} \cdots \sum_{m=0}^{\infty} q^{T(a)+T(b)+\cdots+T(m)}
$$

can be expressed as

$$
\psi(q)^{m}=\sum_{n=0}^{\infty} t_{m}(n) q^{n},
$$

where $t_{m}(n)$ is the number of representations of the integer $n$ by $m$ triangular numbers [42].

The triangular lacunary function is given by Equation (17) by generalizing $q$ to complex variable $z$ : $\psi(z)$. The following theorem showa how the centered polygonal lacunary functions are related to $\psi(z)$.

Theorem 7. For any positive integer $k$,

$$
f^{(k)}(z)=z \psi\left(z^{k}\right)
$$

Proof. Starting from the right hand side of the theorem and using Equation (7), one sees

$$
z \psi\left(z^{k}\right)=z \sum_{n=0}^{\infty}\left(z^{k}\right)^{\frac{n^{2}+n}{2}}=\sum_{n=0}^{\infty} z^{\frac{k n^{2}+k n}{2}+1} .
$$

Shifting the dummy index $n \rightarrow n-1$

$$
z \psi\left(z^{k}\right)=\sum_{n=1}^{\infty} z^{\frac{k n^{2}+k n}{2}+1}=f^{(k)}(z)
$$

The last expression is the centered polygonal lacunary function which completes the proof.

A natural thing to consider about the centered polygonal functions is the behavior of their multiplicative powers. Simply multiplying the functions by themselves $j$ times gives rise to a leading power in the lacunary series to be equal to $j$. It is of interest to place the product on equal footing, in terms of the leading power in the summation, with the original function and with other multiplicative powers. Hence, it is natural to renormalize the multiplicative power by dividing by the appropriate power of $z$ such that the leading term in the lacunary series is to the first power. Figure 6 shows the difference between a non-renormalized power and a renormalized power for the case of $k=3$. In general, the unity level set is more convex for the renormalized case than it is for the non-renormalized case. In both cases, the polished nature of $f^{(k)}(z)$, as defined in [34], persists upon taking powers of the centered polygonal lacunary functions. Invariably, product functions will have associated lacunary series in which the coefficients are not all unity. Consequently, that requirement is suspended for the product functions. 

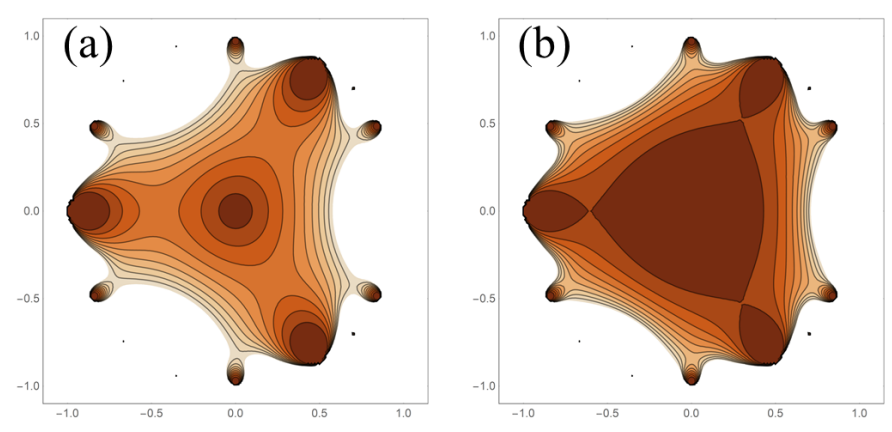

Figure 6. Renormalized (a) and non-renormalized (b) cubic power of $f_{10}^{(k)}$ for $k=3$, i.e., ${ }^{3} R_{10}^{(3)}(z)$ and $\left(f_{10}^{(k)}\right)^{3}$, respectively. In general, renormalization produces a more convex unity level set as compared to the non-renormalized counterpart.

\subsection{Renormalized Powers}

The connection between the triangular numbers and the centered polygonal numbers allows one to utilize the efficiency of the generating functions of $\psi$ in study of centered polygonal lacunary functions. Here, this is used to develop a renormalized power function and a symmetry sequence function.

The renormalized power of a centered polygonal lacunary functions is defined as follows.

Definition 5. Renormalized power. Let $j$ and $k$ be positive integers. The renormalized jth power of $f^{(k)}(z)$ is

$$
{ }^{j} R^{(k)}(z) \equiv \frac{1}{z^{j-1}}\left(f^{(k)}(z)\right)^{j} .
$$

when $f^{(k)}(z)$ is truncated at $N$,

$$
{ }^{j} R_{N}^{(k)}(z) \equiv \frac{1}{z^{j-1}}\left(f_{N}^{(k)}(z)\right)^{j} .
$$

The following theorem is an immediate result of Theorem 7 and Definition 5 .

Theorem 8. Let $j$ and $k$ be positive integers. Then,

$$
{ }^{j} R^{(k)}(z)=z\left(\psi\left(z^{k}\right)\right)^{j}=\sum_{n=0}^{\infty} t_{j}(n) z^{k n+1} .
$$

Proof. From Definition 5 and application of Theorem 7,

$$
\begin{aligned}
j_{R^{(k)}}(z) & =\frac{1}{z^{j-1}}\left(f^{(k)}(z)\right)^{j}=\frac{1}{z^{j-1}}\left(z\left(\psi\left(z^{k}\right)\right)^{j}\right. \\
& =z\left(\psi\left(z^{k}\right)\right)^{j} .
\end{aligned}
$$

The final relation follows from the summation form of $\psi$.

A subtle aspect of Theorem 8 is that the coefficients are decoupled from the powers. This is a manifestation of the important property of the centered polygonal lacunary functions that many of their characteristics are independent of $k$. The same is true here. One need only find the $t_{j}(n)$ and one has the whole family of ${ }^{j} R^{(k)}(z)$. The case of $k=3$ is shown in Figure 7 . The top left panel shows the original function, whereas the remaining panels shows increasing $j$ values. This example is illustrative of the general property of increasing convexity of the unity level set with increasing $j$. Further, the $k$-fold symmetry is maintained as well as the relative polish (again, see [34]) of the unity level sets. 

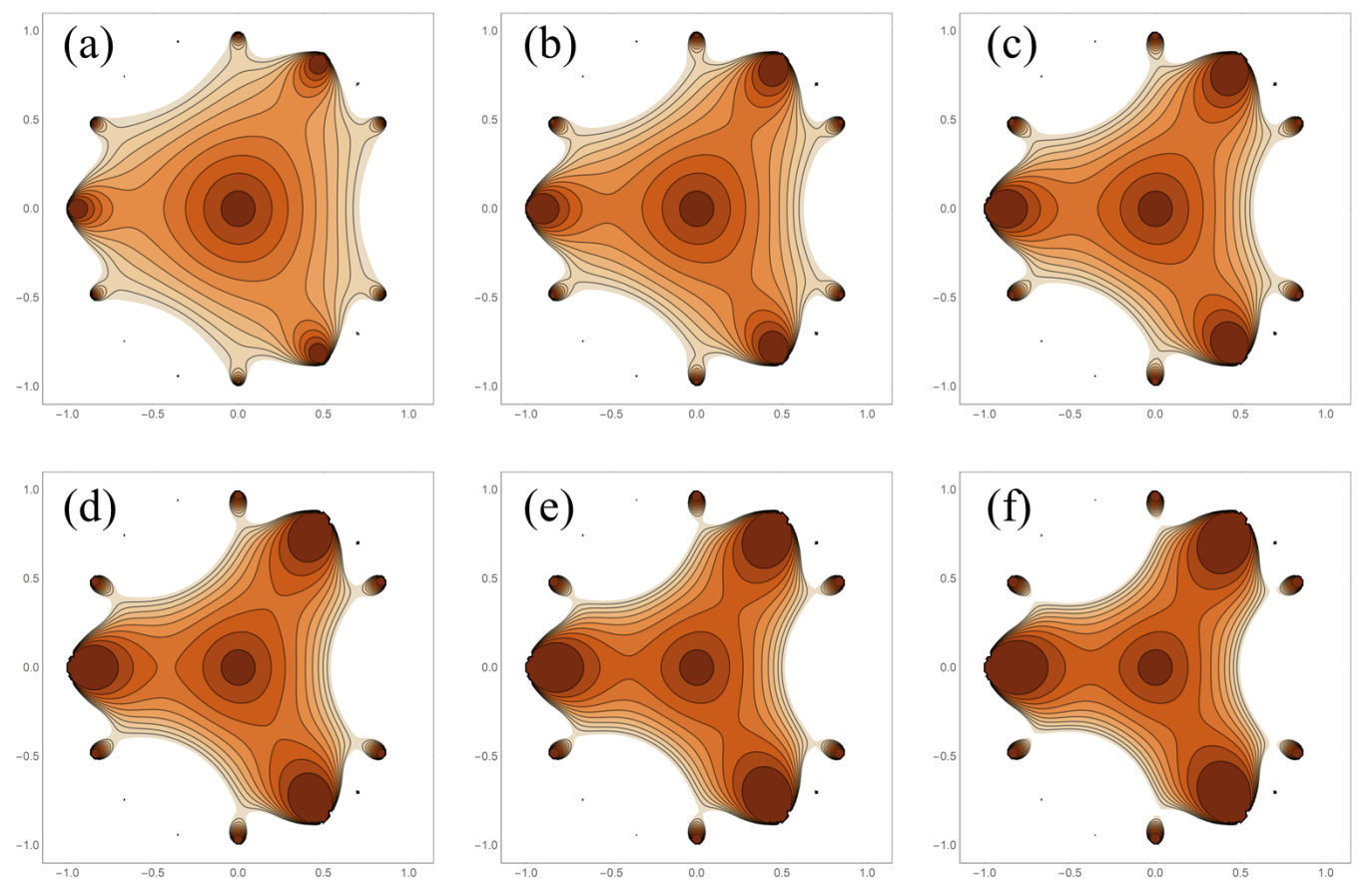

Figure 7. Renormalized powers (first, $j=1$, to sixth, $j=6$ ) of ${ }^{j} R_{10}^{(3)}(z)((\mathbf{a})$ through (f) respectively). Three-fold symmetry is maintained the function narrows in towards the symmetry axes as the power increase, i.e., the convexity of the main unity contour is increased.

\subsection{Symmetry-Sequence Products}

In addition to multiplicative powers, it is equally natural to simply multiply two different centered polygonal lacunary functions. This certainly can be done but it will, in general, break the $k$-fold rotational symmetry. An exception to this is the special case when an increasing sequence of $k$ values are chosen such that each larger $k$ is a multiple of the the base $k$ value. For example, the product $\frac{1}{z^{23}} \prod_{j=1}^{24} f_{10}^{(3 j)}(z)$ is one of these special sequences. For these types of products, the $k$-fold rotational symmetry is maintained. This particular function is shown in Figure 8. An elaborate and rather beautiful unity level set is seen. The presence of the higher $k$ factors in the product are responsible for this. Strong convexity is seen at the angles $\phi=0, \frac{2 \pi}{3}, \frac{4 \pi}{3}$.

The higher rotational symmetry factors $M=2,3, \ldots$ give rise to more structure in the function. Figure 9 shows the case of $k=1$ and $M$ increasing by 1 from 1 to 6 .

Definition 6. Renormalized symmetry-sequence product. The k-based renormalized symmetry-sequence product of $f^{(k)}(z)$ is

$$
{ }^{M_{K}}{ }^{(k)}(z) \equiv \frac{1}{z^{M-1}} \prod_{j=1}^{M} f^{(j k)}(z) .
$$

when $f^{(k)}(z)$ is truncated at $N$,

$$
{ }^{M} K_{N}^{(k)}(z) \equiv \frac{1}{z^{M-1}} \prod_{j=1}^{M} f_{N}^{(j k)}(z)
$$

\section{Theorem 9.}

$$
M_{K}(k)(z)=\sum_{a=0}^{\infty} \sum_{b=0}^{\infty} \cdots \sum_{c=0}^{\infty} t_{M}(a) t_{M-1}(b) \cdots t_{1}(c) z^{k(a+b+\cdots+c)+1}
$$


Proof. Beginning with Definition 6,

$$
\begin{aligned}
{ }^{M_{K}}{ }^{(k)}(z) & =\frac{1}{z^{M-1}} \prod_{j=1}^{M} f^{(j k)}(z) \\
& =\frac{1}{z^{M-1}} f^{(k)}(z) f^{(2 k)}(z) \cdots f^{(M k)}(z) .
\end{aligned}
$$

Now, using Theorem 7 , the $f^{(j k)}(z)$ can be written in terms of $\psi$ as

$$
M_{K}^{(k)}(z)=\frac{1}{z^{M-1}} z^{M} \psi\left(z^{k}\right) \psi\left(z^{2 k}\right) \cdots \psi\left(z^{M k}\right) .
$$

Now, expressing the $\psi$ in their summation form gives

$$
M_{K}(k)(z)=z \sum_{n_{1}=0}^{\infty} z^{k T\left(n_{1}\right)} \sum_{n_{2}=0}^{\infty} z^{2 k T\left(n_{2}\right)} \ldots \sum_{n_{3}=0}^{\infty} z^{M k T\left(n_{3}\right)} .
$$

Consider the $j$ th factor,

$$
\sum_{n_{j}=0}^{\infty} z^{j k T\left(n_{3}\right)}=\sum_{n_{j}=0}^{\infty} z^{k T\left(n_{3}\right)} z^{k T\left(n_{3}\right)} \cdots z^{k T\left(n_{3}\right)} .
$$

Combined with the presence of the other factors, one gets powers of $\psi, 1$ through $M$. Then, by rearranging as in Equation (19) for each power 1 through $M$ and factoring in the external factor of $z$, one gets the final result

$$
M_{K}(k)(z)=\sum_{a=0}^{\infty} \sum_{b=0}^{\infty} \cdots \sum_{c=0}^{\infty} t_{M}(a) t_{M-1}(b) \cdots t_{1}(c) z^{k(a+b+\cdots+c)+1}
$$

and the proof is completed.

As long as $M \geq 3$, all possible $k n+1$ exponents will appear and the above summation can be written more succinctly as

$$
M_{K}(k)(z)=\sum_{n=0}^{\infty} s_{M}(n) z^{k n+1}
$$

albeit $s_{M}(n)$ is rather complicated. In fact,

$$
s_{M}(n)=\sum_{\pi_{n}} t_{M}\left(n_{1}\right) t_{M-1}\left(n_{2}\right) \cdots t_{1}\left(n_{M}\right)
$$

where $\pi_{n}$ is interpreted as one of the ordered integer partitions of $n$. 


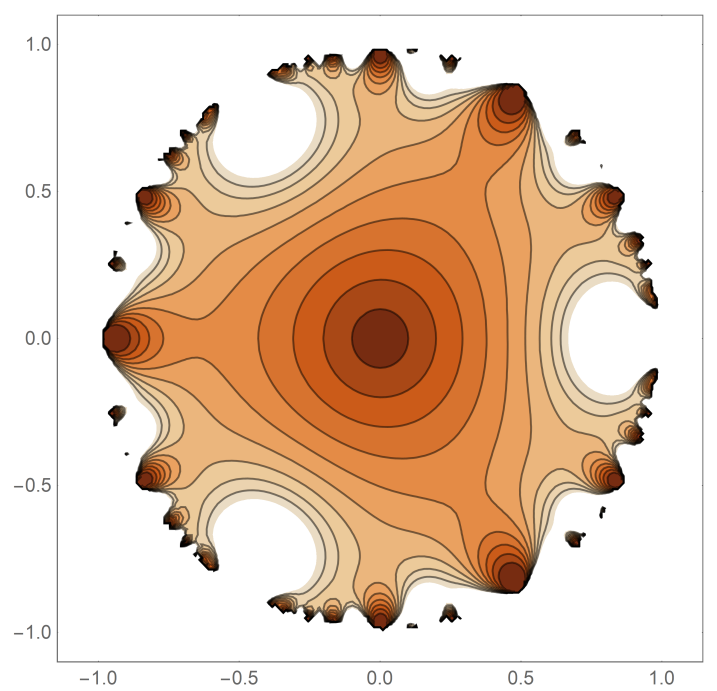

Figure 8. The product of the sequence of functions ${ }^{23} K_{10}^{(3)}(z)$. The three-fold rotational symmetry is maintained in this case, although the structure of the unity level set becomes more elaborate.
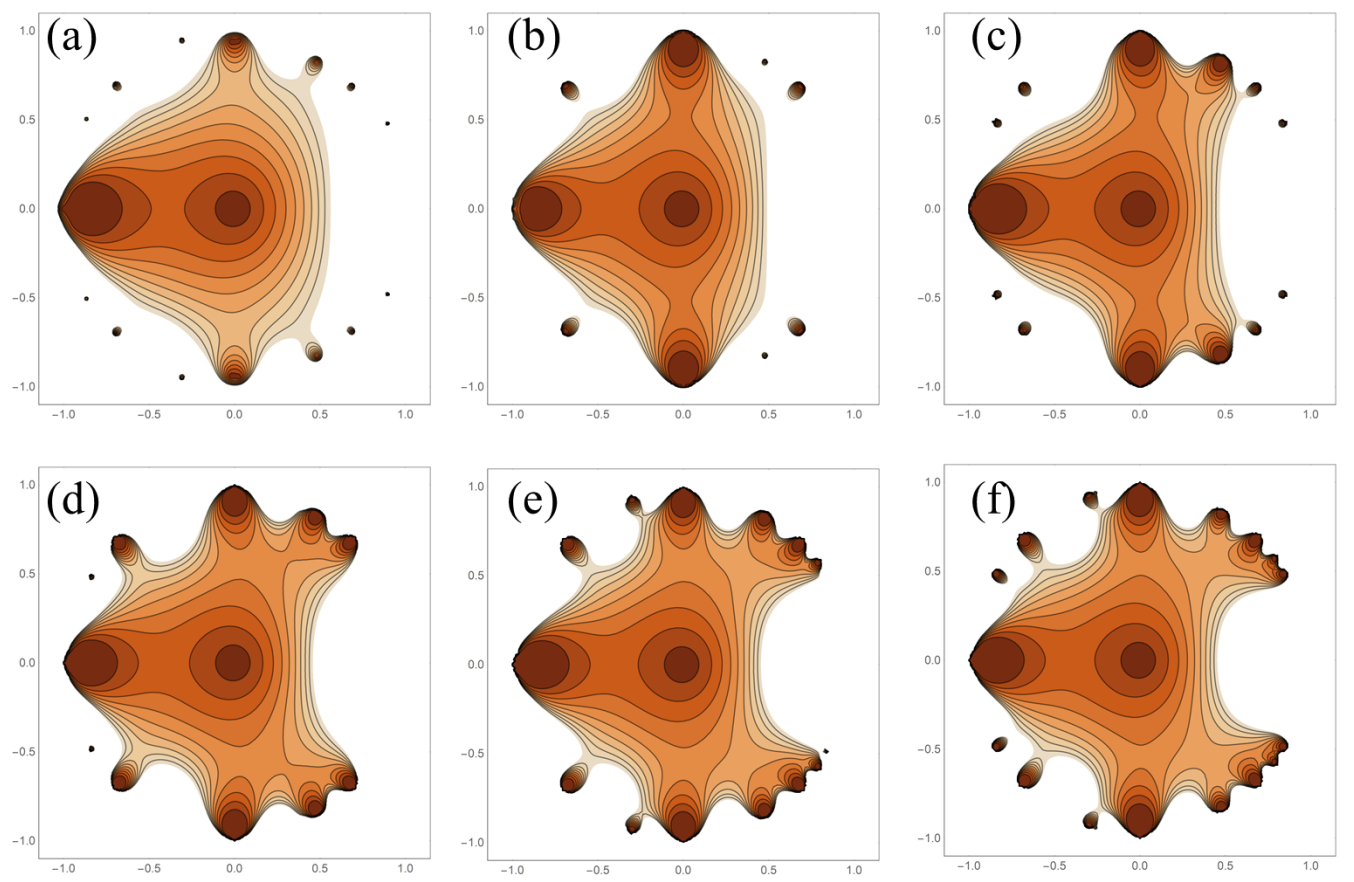

Figure 9. The product of the sequence of functions ${ }^{{ }^{M}} K_{10}^{(1)}(z)$ for $M=1$ through $M=6((\mathbf{a})$ through (f) respectively).

\section{Conclusions}

One focus of this work is the rotationally symmetric lacunary functions. This work builds upon previous studies of centered polygonal lacunary functions [34-36] by adding to the literature two theorems regarding symmetry of these functions. The proven theorems show rotational and dihedral mirror symmetry carried by the centered polygonal lacunary functions at the modulus level. Beyond this, the theorems provide a general framework for constructing other lacunary functions that exhibit the same symmetries. Two additional theorems are given in this regard. This analysis enables a better explorations of the effects of the gap behavior on the qualitative features of the associated lacunary functions. By way of example, Fibonacci-based and power-based lacunary functions are 
given. Additionally, a parameterized, superlinear function allows for a more systematic study of the effects of gap behavior on the shape of the main unity contour. Fast acceleration of the underlying sequence given by $g(n)$ gives low convexity while slow acceleration yields high convexity.

One of the general features of functions related to the lacunary functions studied in previous work is that of self-similarity and scale-invariance $[5,6,20,34,36]$. It may be the case that the knowledge of how to construct rotationally symmetric lacunary functions can be another tool in renormalization procedures.

Another focus of this work is the study of the behavior two types of products of centered polygonal lacunary functions, ${ }^{j} R^{(k)}(z)$ and ${ }^{M} K^{(k)}(z)$. These functions exhibit the same rotational and dihedral mirror symmetry as the base centered polygonal lacunary functions.

Some obvious areas for further study include generalizing Equation (1) to include non-unity coefficients, considering some of the numerous known sequences in addition to the Fibonacci and centered polygonal sequences, and continuing to try to connect to applications in physics and engineering. Potential areas of application in physics are to the partition function of statistical mechanics, nonlinear phase chirp in optics, and iterative dynamical systems.

Author Contributions: L.K.M., K.S., T.V., and D.J.U. conceived of and designed the investigation; K.S. and D.J.U. provided background for the investigation; D.J.U. wrote the MATHEMATICA code to perform the investigation; L.K.M., K.S., T.V., and D.J.U analyzed the data; D.J.U. wrote the original draft of manuscript; and L.K.M., K.S., T.V., and D.J.U. edited the manuscript. All authors have read and agreed to the published version of the manuscript.

Funding: This research was funded by the Concordia College Chemistry Endowment Fund.

Acknowledgments: Douglas R. Anderson, and Drew Rutherford are acknowledged for valuable discussion.

Conflicts of Interest: The authors declare no conflict of interest. The funders had no role in the design of the study; in the collection, analyses, or interpretation of data; in the writing of the manuscript, or in the decision to publish the results.

\section{References}

1. Hille, E. Analytic Function Theory, Volume I; Ginn and Company: Boston, MA, USA, 1959.

2. Hille, E. Analytic Function Theory, Volume II; Ginn and Company: Boston, MA, USA, 1962.

3. Shudo, A.; Ikeda, K.S. Tunneling effect and the natural boundary of invariant tori. Phys. Rev. Lett. 2012, 109, 154102. [CrossRef] [PubMed]

4. Creagh, S.C.; White, M.M. Evanescent escape from the dielectric ellipse. J. Phys. A 2010, 43, 465102. [CrossRef]

5. Wölk, S.; Merkel, W.; Schleich, W.P.; Averbukh, I.S.; Girard, B. Factorization of numbers with Gauss sums: I. Mathematical background. New J. Phys. 2011, 13, 103007. [CrossRef]

6. Schleich, W.P. Quantum Physics and Number Theory; Institut für Quantenphysik, Universität Ulm. Available online: ncp.edu.pk/docs/iscp/quantum_physics_and_number_theory_w.schleich_islamabad.pdf (accessed on 25 May 2020).

7. Greene, J.M.; Percival, I.C. Hamiltonian maps in the complex plane. Phys. 3D 1981, 3, 530-548.

8. Nickel, B. On the singularity structure of the 2D Ising model susceptibility. J. Phys. A Math. Gen. 1999, 32, 3889-3906. [CrossRef]

9. Guttmann, A.J.; Enting, I.G. Solvability of some statistical mechanical systems. Phys. Rev. Lett. 1996, 76, 344-347. [CrossRef]

10. Orrick, W.P.; Nickel, B.G.; Guttmann, A.J.; Perk, J.H.H. Critical behavior of the two-dimensional Ising susceptibility. Phys. Rev. Lett. 2001, 86, 4120-4123. [CrossRef]

11. Jensen, G.; Pommerenke, C.; Ramirez, J.M. On the path properties of a lacunary power series. Proc. Am. Math. Soc. 2014, 142, 1591-1606. [CrossRef]

12. Yamada, H.S.; Ikeda, K.S. Analyticity of quantum states in one-dimen-sional tight-binding model. Eur. Phys. J. B 2014, 87, 208. [CrossRef]

13. Salem, P.; Zygmund, A. On lacunary trigonometric series. Proc. Natl. Acad. Sci. USA 1947, 33, $333-338$. [CrossRef]

14. Katznelson, Y. An Introduction to Harmonic Analysis; Dover Publications: New York, NY, USA, 1976. 
15. Aistleitner, C.; Berkes, I. On the central limit theorems for $f\left(n_{k} x\right)$. Probab. Theory Relat. Fields 2010, 146, 267-289. [CrossRef]

16. Fukuyama, K.; Takahashi, S. The central limit theorem for lacunary series. Proc. Am. Math. Soc. 1999, 127, 599-608. [CrossRef]

17. Kahane, J.-P. A century of interplay between Taylor series, Fourier series and Brownian motion. Bull. Lond. Math. Soc. 1997, 29, 257-279. [CrossRef]

18. Blendeck, T.; Michaliček, J. $L^{1}$-norm estimates of character sums defined in a Sidom set in the dual of a Kac algebra. J. Oper. Theory 2013, 70, 375-399. [CrossRef]

19. Wang, S. Lacunary Fourier series for compact quantum groups. Comm. Math. Phys. 2017, 349, 895-945. [CrossRef]

20. Costin, O.; Huang, M. Behavior of lacunary series at the natural boundary. Adv. Math. 2009, 222, 1370-1404. [CrossRef]

21. Boyd, J.P. The breakdown of Darboux's principle and natural boundaries for a function periodised from a Ramanujan Fourier transform pair. East Asian J. Appl. Math. 2019, 9, 409-423. [CrossRef]

22. Dahlqvist, P. On the effect of pruning on the singularity structure of zeta functions. J. Math. Phys. 1997, 38, 4273. [CrossRef]

23. Patkowski, A.E. A family of lacunary partition functions. New Zealand J. Math. 2008, 38, 87-91.

24. Lovejoy, J. Lacunary partition functions. Math. Res. Lett. 2002, 9, 191-198. [CrossRef]

25. Lovejoy, J. More lacunary partition functions. Illinois J. Math. 2003, 47, 769-773. [CrossRef]

26. Ono, K.; Robbins, S.; Wahl, P.T.; On the representation of integers as sums of triangular numbers. Aequ. Math. 1995, 50, 73-94. [CrossRef]

27. Sebbar, A. On two lacunary series and modular curves. In The Mathematical Legacy of Leon Ehrenpresis; Sabadin, I., Struppa, D.C., Eds.; Springer: Mailand, Italy, 2012.

28. Lovejoy, J.; Osburn, R. The Bailey chain and mock theta functions. Adv. Math. 2013, 238, 442-458. [CrossRef]

29. Kayumov, I.R.; Ponnusamy, S. Bohr's inequalities for the analytic functions with lacunary series and harmonic functions. J. Math. Anal. Appl. 2018, 465, 857-871. [CrossRef]

30. Liu, X.; Liu, T. The Bohr inequality for holomorphic mappings with lacunary series in several complex variables. J. Math. Anal. Appl. 2020, 485, 123844. [CrossRef]

31. Eckstein, M.; Zając A. Asymptotic and exact expansion of heat traces. Math. Phys. Anal. Geom. 2015 18, 28. [CrossRef]

32. Behr, N.; Dattoli, G.; Duchamp, G.H.E.; Licciardi, S.; Penson K.A. Operational methods in the study of Sobolev-Jacobi polynomials. Mathematics 2019, 7, 124. [CrossRef]

33. Kişi, Ö.; Gümüs, H., Savas, E. New definitions about $A^{\mathcal{I}}$-statistical convergence with respect to a sequence of modulus functions and lacunary sequences. Axioms 2018, 7, 24.

34. Sullivan, K.; Rutherford, D.; Ulness, D.J. Centered polygonal lacunary sequences. Mathematics 2019, 7, 943. [CrossRef]

35. Sullivan, K.; Rutherford, D.; Ulness, D.J. Centered polygonal lacunary Graphs: A graph theoretic approach to p-sequences of centered polygonal lacunary functions. Mathematics 2019, 7, 1021. [CrossRef]

36. Mork, L.K.; Vogt, T.; Sullivan, K.; Rutherford, D.; Ulness, D.J. Exploration of filled-in Julia sets arising from centered polygonal lacunary functions. Fract. Fract. 2019, 3, 42. [CrossRef]

37. Mork, L.K.; Sullivan, K.; Ulness, D.J. Taming the natural boundary of centered polygonal lacunary functions: Restriction to the symmetry angle space. Mathematics 2020, 8, 568. [CrossRef]

38. Schlicker, S.J. Numbers simultaneously polygonal and centered polygonal. Math. Mag. 2011, 84, 339-350. [CrossRef]

39. Teo, B.K.; Sloane, J.A. Magic numbers in polygonal clusters. Inorg. Chem. 1985, 24, 4545-4558. [CrossRef]

40. Deza, E.; Deza, M.-M. Figurate Numbers; World Scientific: Hackensack, NJ, USA, 2012.

41. Edgar, T. Visual decompositions of polygonal number. College Math. J. 2020, 51, 9-12. [CrossRef]

42. Hirschhorn, M.D.; Sellers, J.A. Partitions into three triangular numbers. Aust. J. Comb. 2004 30, 301-318.

(C) 2020 by the authors. Licensee MDPI, Basel, Switzerland. This article is an open access article distributed under the terms and conditions of the Creative Commons Attribution (CC BY) license (http:/ / creativecommons.org/licenses/by/4.0/). 